\title{
ALMOST LOCALLY POLYHEDRAL CURVES IN EUCLIDEAN $n$-SPACE
}

\author{
BY
}

J. C. CANTRELL AND C. H. EDWARDS, JR.(1)

Fox and Artin [2] have given examples of wild arcs and curves in $E^{3}$ which fail to be locally polyhedral at only one or two points. It is shown in this paper that no such "simple" examples of wild curves are to be expected in dimensions higher than three. In particular, it is proved that a wild simple closed curve in Euclidean $n$-space $E^{n}, n>3$, must fail to be locally polyhedral at each point of a Cantor set. Examples of such wild curves in $E^{n}$ have been given by Blankinship [1].

A set $K$ in $E^{n}$ is called tame if there is a homeomorphism $h$ of $E^{n}$ onto itself such that $h(K)$ is polyhedral (relative to the standard triangulation of $\left.E^{n}\right)$. Otherwise $K$ is wild. $K$ is said to be locally polyhedral at the point $p \in K$ if there exists a neighborhood $N$ of $p$ such that $\mathrm{Cl}(N \cap K)$ is a polyhedron. The map $h: E^{n} \rightarrow E^{n}$ is said to be locally semilinear at $x$ if there is a neighborhood $N$ of $x$ such that $h \mid N$ is semilinear.

In this paper, $S(p, \varepsilon)$ denotes the set of points $x \in E^{n}$ whose distance $\rho(x, p)$ from $p$ is less than $\varepsilon$.

The local connectivity of an arc gives the following lemma.

Lemma 1. Suppose that $A$ is an arc in $E^{n}$ with $p$ an interior point of $A$. Given $\varepsilon>0$, there exists $\delta>0$ such that, if $L$ is any subarc of $A$ whose endpoints lie in $S(p, \delta)$, then $L \subset S(p, \varepsilon)$.

Lemma $2\left({ }^{2}\right)$. Suppose that $C$ is a simple closed curve in $E^{n}, n>3$, and that $B$ is the set of points at which $C$ fails to be locally polyhedral. If $p$ is an isolated point of $B$, then, given $\varepsilon>0$, there exists a homeomorphism $h$ of $E^{n}$ onto $E^{n}$ such that

(a) $h$ is the identity on $E^{n}-S(p, \varepsilon)$,

(b) $h$ is locally semilinear except at $p$,

(c) $h(C)$ is locally polyhedral at $h(p)$.

Presented to the Society, January 22, 1962; received by the editors July 9, 1962.

(1) This research was supported in part by the National Science Foundation, Grants 8239 and 11665 .

(2) The authors are indebted to Professor O. G. Harrold for suggesting the Problem solved in Lemma 2. 
Proof. Let $q_{1}$ and $q_{2}$ be two points of $C-S(p, \varepsilon)$ and let $L_{1}, L_{2}, L_{3}$ be the three subarcs of $C$ with endpoints $q_{1}$ and $p, q_{2}$ and $p, q_{1}$ and $q_{2}$, respectively, such that $C=L_{1} \cup L_{2} \cup L_{3}$ and each subarc meets either of the other subarcs in a single endpoint.

Let $\varepsilon_{1}=\varepsilon$ and let $N_{1}$ be the closed cubical neighborhood of $p$ of diameter $\varepsilon_{1}$. Let $\delta_{1}$ be given by Lemma 1 for $\varepsilon_{1} / 3, p$, and $A=L_{1} \cup L_{2}$. Let $\varepsilon_{2}=\min \left[\delta_{1}, \rho\left(p, L_{3}\right)\right]$ and let $N_{2}$ be the closed cubical neighborhood of $p$ of diameter $\varepsilon_{2}$. For $i>2$ let $\delta_{i-1}$ be given by Lemma 1 for $\varepsilon_{i-1} / 3, p$, and $A=L_{1} \cup L_{2}$. Let $\varepsilon_{i}=\delta_{i-1}$ and let $N_{i}$ be the closed cubical neighborhood of $p$ of diameter $\varepsilon_{i}$.

By making use of a semilinear deformation in a small neighborhood of $\operatorname{Bd} N_{2 i}$, if necessary, we may assume that $C \cap \mathrm{Bd} N_{2 i}$ is a finite set of points, and that no pair of components of $C-N_{2 i}$ share a common endpoint. For each positive integer $i$, let $u_{i 1}, \cdots, u_{i k(i)}$ be the closures of those components of $C-N_{2 i}$ which have both endpoints on $\mathrm{Bd} N_{2 i}$. Observe that each of these sets is contained in the half open annulus Int $N_{2 i-1}-$ Int $N_{2 i}$. Let $w_{i 1}$ be a polyhedral arc in $\operatorname{Bd} N_{2 i}$ which connects the endpoints of $u_{i 1}$ and, except for those two points, is disjoint from $C$. The resulting simple closed curve $d_{i 1}=u_{i 1} \cup w_{i 1}$ bounds a polyhedral 2-cell $D_{i 1}$ in Int $N_{2 i-1}$, since $n>3$ [3, p. 32]. Hence we may assume that $D_{i 1}$ intersects $C$ only in $w_{i 1}$. We may further assume that $D_{i 1}$ and $\mathrm{Bd} N_{2 i}$ are in relative general position, so that each component of Int $D_{i 1} \cap \mathrm{Bd} N_{2 i}$ is either a simple closed curve or an open arc (whose closure may be either an arc intersecting $w_{i 1}$ in its endpoints or a simple closed curve intersecting $w_{i 1}$ in a single point).

Let $e$ be $a$ simple closed curve component of Int $D_{i 1} \cap \mathrm{Bd} N_{2 i}$ which contains no other such component in its interior (relative to $D_{i 1}$ ). Let $Y$ be the subdisk of $D_{i 1}$ bounded by $e$. Let $E$ and $F$ be the components of $E^{n}-\mathrm{Bd} N_{2 i}$ and labeled so that $Y \subset \mathrm{Cl} E$.

Let $r$ be a point in $F$, sufficiently close to $\mathrm{Bd} N_{2 i}$ for $X$ (the join of $r$ and $e$ ) to meet $D_{i 1} \cup C$ only in $e$. Define $D_{i 1}^{\prime}=\left(D_{i 1}-Y\right) \cup X$ and deform $D_{i 1}^{\prime}$ semilinearly away from $\mathrm{Bd} N_{2 i}$ in a sufficiently small neighborhood of $e$ so that no new intersections are introduced. The disk $D_{i 1}^{\prime \prime}$ thus obtained is bounded by $d_{i 1}$ and intersects $\mathrm{Bd} N_{2 i}$ in exactly those components, other than $e$, in which $D_{i 1}$ intersected Bd $N_{2 i}$. Since the open arc components of Int $D_{i 1} \cap \mathrm{Bd} N_{2 i}$ can be eliminated by a similar process of exchanging disks, after a finite number of steps we obtain a disk $D_{i 1}^{*}$ which, except for $w_{i 1}$, is contained in the open annulus $\operatorname{Int}\left(N_{2 i-1}-N_{2 i}\right)$ and intersects $C$ only in $u_{i 1}$.

Let $\eta>0$ be such that the $\eta$-neighborhood $S_{i 1}$ of $D_{i 1}^{*}$ is contained in Int $\left(N_{2 i-1}-N_{2 i+1}\right)$ and intersects $C-N_{2 i}$ only in $u_{i 1}$. By a sequence of simplicial moves across the 2-simplexes of $D_{i 1}^{*}$ the arc $u_{i 1}$ may be moved onto the arc $w_{i 1}$. By making use of a corresponding space homeomorphism [5, Lemma 3], we may deform $u_{i 1}$ onto $w_{i 1}$ and then into Int $N_{2 i}$ by a semilinear space homeomorphism which is the identity outside $S_{i 1}$.

The components $u_{i 2}, \cdots, u_{i k(i)}$ are successively moved into $\operatorname{Int}\left(N_{2 i}-N_{2 i+1}\right)$ by 
a technique similar to that used on $u_{i 1}$. We are careful in each move to leave the remaining components fixed. This is to keep from introducing new intersections with $\mathrm{Bd} N_{2 i}$. We denote the composition of these moves by $f_{i}$ and observe that $f_{i}$ is a semilinear space homeomorphism which is fixed outside Int $\left(N_{2 i-1}-N_{2 i+1}\right)$. Also, if $a_{i}$ is the first point of $L_{1} \cap \mathrm{Bd} N_{2 i}$ relative to the order of $L_{1}$ from $q_{1}$ to $p$ and if $b_{i}$ is the first point of $L_{2} \cap \mathrm{Bd} N_{2 i}$ relative to the order of $L_{2}$ from $q_{2}$ to $p$, then $f_{i}(C) \cap \operatorname{Bd} N_{2 i}=a_{i} \cup b_{i}$.

We define a mapping $f$ of $E^{n}$ onto $E^{n}$ by the equations

$$
\begin{aligned}
& f(x)=x \quad \text { if } x \in E^{n}-N_{1}, \\
& f(x)=f_{i}(x) \text { if } x \in N_{2 i-1}-N_{2 i+1}, \quad i=1,2, \cdots, \\
& f(p)=p .
\end{aligned}
$$

It is clear since, for each $i, f_{i}$ is fixed on $\mathrm{Bd} N_{2 i-1} \cup \operatorname{Bd} N_{2 i+1}$ and $f_{i}$ eliminates all but two points of intersection of $C$ and $\mathrm{Bd} N_{2 i}$, that $f$ is a homeomorphism, semilinear except at $p$, and that $f(C) \cap \operatorname{Bd} N_{2 i}=a_{i} \cup b_{i}, i=1,2, \cdots$.

We now consider the curve $f(C)$. Let $L_{1 i}=f\left(L_{1}\right) \cap \mathrm{Cl}\left(N_{2 i}-N_{2 i+2}\right)$ and $L_{2 i}=f\left(L_{2}\right) \cap \mathrm{Cl}\left(N_{2 i}-N_{2 i+2}\right)$. Let $x_{i}$ be the point of intersection of the linear segment $\overline{a_{1} p}$ with $\mathrm{Bd} N_{2 i}$ and let $y_{i}$ be the point of intersection of $\overline{b_{1} p}$ with Bd $N_{2 i}$. Let $\phi_{i}$ be a semilinear space homeomorphism which is fixed outside $N_{2 i-1}-N_{2 i+1}$ and which carries $\operatorname{Bd} N_{2 i}$ onto $\operatorname{Bd} N_{2 i}$, with $\phi_{i}\left(a_{i}\right)=x_{i}$ and $\phi_{i}\left(b_{i}\right)=y_{i}$. Since $a_{1}=x_{1}$ and $b_{1}=y_{1}$, we will assume that $\phi_{1}$ is the identity homeomorphism. The simple closed curve $\overline{x_{i} x_{i+1}} \cup \phi_{i+1} \phi_{i}\left(L_{1 i}\right)$ bounds a polyhedral disk $D_{i}$, which may be taken to be disjoint from $\overline{y_{i} y_{i+1}} \cup \phi_{i+1} \phi_{i}\left(L_{2 i}\right)$. Furthermore, in the light of the elimination o composent scheme $\mathrm{u}$ ed atove, $D_{i}$ may be selected so that $D_{i} \cap\left(\operatorname{Bd} N_{2 i} \cup \operatorname{Bd} N_{2 i+2}\right)=x_{i} \cup x_{i+1}$. The arc $\phi_{i+1} \phi_{i}\left(L_{1 i}\right)$ is then moved across the disk $D_{i}$ onto the arc $\overline{x_{i} x_{i+1}}$ by a semilinear space homeomorphism $\psi_{i 1}$, which is the identity outside $N_{2 i}-N_{2 i+2}$ and on $\phi_{i+1} \phi_{i}\left(L_{2 i}\right)$. Similarly $\phi_{i+1} \phi_{i}\left(L_{2 i}\right)$ is moved onto $\overline{y_{i}} \bar{y}_{i+1}$ by a space homeomorphism $\psi_{i 2}$, which is fixed outside $N_{2 i}-N_{2 i+2}$ and on $\bar{x}_{i} x_{i+1}$. The composition $\psi_{i 2} \psi_{i 1}$ is denoted by $\psi_{i}$

A mapping $g$ of $E^{n}$ onto $E^{n}$ is defined by the equations

$$
\begin{aligned}
& g(x)=x \quad \text { if } x \in E^{n}-N_{2}, \\
& g(x)=\psi_{i} \phi_{i+1} \phi_{i}(x) \text { if } x \in N_{2 i}-N_{2 i+2} \quad i=1,2, \cdots, \\
& g(p)=p .
\end{aligned}
$$

The fact that $\psi_{i} \phi_{i+1} \phi_{i}$ and $\psi_{i+1} \phi_{i+2} \phi_{i+1}$ agree on $\mathrm{Bd} N_{2 i+2}$ (each reduces to $\left.\phi_{i+1}\right)$ insures that $g$ is a homeomorphism. It is clear that $g$ is semilinear except at $p$ and that the subarc of $f\left(L_{1}\right)$ from $a_{1}$ to $p$ is carried onto the segment $\overline{a_{1}} \bar{p}$ and that the subarc of $f\left(L_{2}\right)$ from $b_{1}$ to $p$ is carried onto the segment $\overline{b_{1} p \text {. }}$

Finally the desired homeomorphism $h$ is taken to be the composition $h=g f$, so that the proof of Lemma 2 is complete. 
Notice that the essential point upon which the proof of Lemma 2 depends is the fact that polyhedral simple closed curves cannot knot or link in $E^{n}$ if $n>3$, whereas the construction of the typical example of a wild curve in $E^{3}$, locally polyhedral except at a single point, involves knotting or linking in a neighborhood of the exceptional point.

The following lemma will serve as the first step in an inductive proof of the principal theorem.

LEMMA 3. If $C$ is a simple closed curve in $E^{n}, n>3$, denote by $B$ the set of points at which $C$ fails to be locally polyhedral, and by $B^{\prime}$ the derived set of $B$. Then, given $\varepsilon>0$ and a compact set $F$ not meeting $E=B-B^{\prime}$, there is is a homeomorphism $h$ of $E^{n}$ onto itself such that

(a) $h(x)=x$ if $x \in B^{\prime} \cup\left[E^{n}-S(B, \varepsilon)\right]$,

(b) $h$ is locally semilinear on $E^{n}-B$,

(c) $h(C)$ is locally polyhedral at each point of $h(C)-B^{\prime}$,

(d) $\rho(x, h(x)) \leqq \min \{\varepsilon, \varepsilon \cdot \rho(x, F)\}$ for each $x \in E^{n}$.

Proof. Since the discrete set $E$ is at most countable [4, p. 62] enumerate its points in a sequence $\left\{a_{i}\right\}_{1}^{\infty}$ (assume that $E$ is infinite since otherwise Lemma 3 follows immediately from Lemma 2 ). Choose a sequence $\left\{U_{i}\right\}_{1}^{\infty}$ of mutually disjoint open sets with $a_{i} \in U_{i}, U_{i} \cap B^{\prime}=\square$, and diam $U_{i}<\left\{\varepsilon \cdot \rho\left(U_{i}, F\right)\right\}$ for each $i$, and a monotone decreasing sequence $\left\{\varepsilon_{\imath}\right\}_{1}^{\infty}$ of positive numbers with each $\varepsilon_{i}<\varepsilon / 2$ and $\lim _{i \rightarrow \infty} \varepsilon_{i}=0$, such that $\operatorname{Cl} S\left(a_{i}, \varepsilon_{i}\right) \subset U_{i}$ for each $i$.

Using Lemma 2, choose for each $i=1,2, \cdots$ a homeomorphism $h_{i}$ of $E^{n}$ onto itself such that $h_{i}(x)=x$ if $x \in E^{n}-S\left(a_{i}, \varepsilon_{i}\right), h_{i}$ is locally semilinear except at $a_{i}$, and $h_{i}(C)$ is locally polyhedral at $h_{i}\left(a_{i}\right)$.

Then define

$$
h(x)=\left\{\begin{array}{l}
x \quad \text { if } x \in E^{n}-\bigcup_{i=1}^{\infty} S\left(a_{i}, \varepsilon_{i}\right), \\
h_{i}(x) \text { if } x \in S\left(a_{i}, \varepsilon_{i}\right) .
\end{array}\right.
$$

Obviously $h(x)=x$ if $x \in B^{\prime} \cup\left[E^{n}-S(B, \varepsilon)\right]$ and the fact that diam $U_{i}$ $<\varepsilon \cdot \rho\left(U_{i}, F\right)$ for each $i$ implies that $\rho(x, h(x)) \leqq \min \{\varepsilon, \varepsilon \cdot \rho(x, F)\}$ for every $x \in E^{n}$. Since $h_{i} \mid S\left(a_{i}, \varepsilon_{i}\right)$ is $1-1$ onto, it is clear that $h$ is a 1-1 map of $E^{n}$ onto itself. Since $h$ is the identity outside some $n$-cell, to show that $h$ is a homeomorphism it suffices to show that it is continuous.

If $x \in \operatorname{ClS}\left(a_{i}, \varepsilon_{i}\right)$ for some $i$, then there is a neighborhood $U$ of $x$ such that $U \cap S\left(a_{j}, \varepsilon_{j}\right)=\square$ if $i \neq j$. Therefore $h=h_{i}$ on $U$ so that $h$ is continuous at $x$ and is also locally semilinear at $x$, unless $x$ is $a_{i}$.

If $x \in E^{n}-\mathrm{Cl} E-\bigcup_{i=1}^{\infty} \mathrm{Cl} S\left(a_{i}, \varepsilon_{i}\right)$, then there is a neighborhood $V$ of $x$ such that $V \cap \bigcup_{i=1}^{\infty} S\left(a_{i}, \varepsilon_{i}\right)=\square$. To the contrary, suppose that for each positive integer $k$ there is an integer $i_{k}$ such that $S(x, 1 / k) \cap S\left(a_{i_{k}}, \varepsilon_{i_{k}}\right)$ contains a point $x_{k}$. 
Since $x$ is not in any $\operatorname{Cl} S\left(a_{i}, \varepsilon_{i}\right)$, it follows easily that $\lim _{k \rightarrow \infty} i_{k}=\infty$, so that $\lim _{k \rightarrow \infty} \varepsilon_{i_{k}}=0$.

Because $\rho\left(x_{k}, a_{i_{k}}\right)<\varepsilon_{i_{k}}$, while $\lim _{k \rightarrow \infty} x_{k}=x$, it therefore follows that $\lim _{k \rightarrow \infty} a_{i_{k}}=x$, contradicting the fact that $x \notin \mathrm{Cl} E$. Consequently $h$ agrees with the identity in some neighborhood of $x$, so that $h$ is both continuous and locally semilinear at $x$. It is now clear that $h$ is locally semilinear on $E^{n}-B$.

It remains to consider the case of a point $x$ in $\mathrm{Cl} E-\bigcup_{i=1}^{\infty} \mathrm{Cl} S\left(a_{i}, \varepsilon_{i}\right)$. Let $\left\{x_{k}\right\}_{1}^{\infty}$ be an arbitrary sequence of points converging to $x$. Since $h(x)=x$ and $h\left(x_{k}\right)=x_{k}$ if $x_{k} \notin \bigcup_{i=1}^{\infty} S\left(a_{i}, \varepsilon_{i}\right)$, suppose that for each $k$ there is an $i_{k}$ such that $x_{k} \in S\left(a_{i_{k}}, \varepsilon_{i_{k}}\right)$. Since $x$ is not in any $\operatorname{Cl} S\left(a_{i}, \varepsilon_{i}\right)$, it is clear that $\lim _{k \rightarrow \infty} i=\infty$ so that $\lim _{k \rightarrow \infty} \varepsilon_{i_{k}}=0$. Now $h\left(x_{k}\right) \in S\left(a_{i_{k}}, \varepsilon_{i_{k}}\right)$, since $h$ carries $S\left(a_{i_{k}}, \varepsilon_{i_{k}}\right)$ onto itself, so that $\rho\left(x_{k}, h\left(x_{k}\right)\right)<2 \varepsilon_{i_{k}}$. But $\lim _{k \rightarrow \infty} x_{k}=x$ and $\lim _{k \rightarrow \infty} \varepsilon_{i_{k}}=0$. Hence $\lim _{k \rightarrow \infty} h\left(x_{k}\right)=x=h(x)$ so that $h$ is continuous at $x$.

Consequently $h$ is a homeomorphism of $E^{n}$ onto itself. Since it is clear from the construction of $h$ that $h(C)$ is locally polyhedral at each point of $h(C)-B^{\prime}$, this completes the proof of Lemma 3.

THEOREM. Let $C$ be a simple closed curve in $E^{n}, n>3$, and denote by $B_{0}$ the set of points at which $C$ is not locally polyhedral. If $B_{0}$ is countable then $C$ is tame.

Proof. Obviously $B_{0}$ is a compact subset of $C$. Denote by $B_{1}$ the derived set $B_{0}^{\prime}$ of $B_{0}$. Supposing that $B_{\alpha}$ has been defined for every ordinal number $\alpha$ preceding the ordinal number $\beta, B_{\beta}$ is defined as follows: If $\beta$ is an ordinal of the first kind, i.e., $\beta=\alpha+1$ for some $\alpha$, define $B_{\beta}=B_{\alpha}^{\prime}$; while if $\beta$ is an ordinal of the second kind, define $B_{\beta}=\bigcap_{\alpha<\beta} B_{\alpha}$. Since $\left\{B_{\alpha}\right\}$ is a decreasing transfinite sequence of compact subsets of $C$, there exists a first ordinal number $\gamma$ preceding the first uncountable ordinal $\Omega$, such that $B_{\gamma}=B_{\delta}$ for every $\delta>\gamma$ [4, p. 67]. If the compact perfect set $B_{\gamma}$ were nonempty, then it would be uncountable $\left[4\right.$, p. 92], thereby contradicting the hypothesis that $B_{0}$ is countable. Consequently $B_{\gamma}=\square$. It therefore follows that, in order to prove the Theorem, it suffices to show that the following lemma holds for every countable ordinal $\alpha$.

Lemma $A_{\alpha}$. Let $C$ and $B_{0}$ be as in the Theorem. Given a compact set $F$ not intersecting $B_{0}-B_{\alpha}$ and a positive number $\varepsilon$, there is a homeomorphism $h$ of $E^{\prime \prime}$ onto itself such that

(a) $h(x)=x$ if $x \in B_{\alpha} \cup\left[E^{n}-S\left(B_{0}, \varepsilon\right)\right]$,

(b) $h$ is locally semilinear on $E^{n}-B_{0}$,

(c) $h(C)$ is locally polyhedral at each point of $h(C)-B_{\alpha}$,

(d) $\rho(x, h(x)) \leqq \min \{\varepsilon, \varepsilon \cdot \rho(x, F)\}$ for each $x \in E^{n}$.

To prove Lemma $\mathrm{A}_{\alpha}$ for every ordinal number $\alpha$ of the first and second classes, it is sufficient by the transfinite induction principle to show that

(1) Lemma $A_{1}$ is true. 
(2) The truth of Lemma $A_{\alpha}$ implies the truth of Lemma $A_{\alpha+1}$ for every $\alpha<\Omega$.

(3) If $\left\{\alpha_{i}\right\}_{i=1}^{\infty}$ is an increasing sequence of countable ordinals, with $\lim _{i \rightarrow \infty} \alpha_{i}=\alpha_{0}$, such that Lemma $\mathrm{A}_{\alpha_{i}}$ is true for each $i=1,2, \cdots$, then it follows that Lemma $A_{\alpha_{0}}$ is true.

Lemma $A_{1}$ has already been proved as Lemma 3. Now suppose that Lemma $A_{\alpha}$ is true and let $\varepsilon>0$ and a compact set $F$ not meeting $B_{0}-B_{\alpha+1}$ be given. By Lemma $\mathrm{A}_{\alpha}$ there is a homeomorphism $h_{1}$ of $E^{n}$ onto itself such that (a) $h_{1}(x)=x$ if $x \in B_{\alpha} \cup\left[E^{n}-S\left(B_{0}, \varepsilon\right)\right]$, (b) $h_{1}$ is locally semilinear on $E^{n}$ $-B_{0}$, (c) $h_{1}(C)$ is locally polyhedral at each point of $h_{1}(C)-B_{\alpha}$, and (d) $\rho\left(x, h_{1}(x)\right) \leqq \min \{\varepsilon / 2, \varepsilon \cdot \rho(x, F) / 3\}$. Then since the simple closed curve $h_{1}(C)$ is locally polyhedral except at the points of $B_{\alpha}$, by Lemma $A_{1}$ there is a homeomorphism $h_{2}$ of $E^{n}$ onto itself such that (a) $h_{2}(x)=x$ if $x \in B_{\alpha+1} \cup\left[E^{n}-S\left(B_{\alpha}, \varepsilon\right)\right]$, (b) $h_{2}$ is locally semilinear on $E^{n}-B_{\alpha}$, (c) $h_{2} h_{1}(C)$ is locally polyhedral at each point of $h_{2} h_{1}(C)-B_{\alpha+1}$, and (d) $\rho\left(x, h_{2}(x)\right) \leqq \min \{\varepsilon / 2, \varepsilon \cdot \rho(x, F) / 3\}$. It follows that $h=h_{2} h_{1}$ satisfies the requirements of Lemma $A_{\alpha+1}$, so that the truth of Lemma $\mathrm{A}_{\alpha}$ implies the truth of Lemma $\mathrm{A}_{\alpha+1}$.

Now suppose that $\left\{\alpha_{i}\right\}_{1}^{\infty}$ is an increasing sequence of countable ordinals, with $\lim _{i \rightarrow \infty} \alpha_{i}=\alpha_{0}$, such that Lemma $\mathrm{A}_{\alpha_{i}}$ is true for each $i=1,2,3, \cdots$. Let $\varepsilon>0$ and a compact set $F$ not meeting $B_{0}-B_{\alpha_{0}}$ be given. Using Lemma $A_{\alpha_{1}}$, let $h_{1}$ be a homeomorphism of $E^{n}$ onto itself such that (a) $h_{1}(x)=x$ if $x \in B_{a_{1}}$ $\cup\left[E^{n}-S\left(B_{0}, \varepsilon / 2\right)\right]$, (b) $h_{1}$ is locally semilinear on $E^{n}-B_{0}$, (c) $h_{1}(C)$ is locally polyhedral at each point of $h_{1}(C)-B_{\alpha_{1}}$, and (d) $\rho\left(x, h_{1}(x)\right) \leqq \min \{\varepsilon / 2, \varepsilon \cdot \rho(x, F) / 3$, $\left.\left.\rho\left(x, B_{\alpha_{0}}\right) / 4\right)\right\}$. In general, having defined $h_{1}, h_{2}, \cdots, h_{j-1}$ such that $h_{i-1} \cdots h_{2} h_{1}(C)$ is locally polyhedral except possibly at the points of $B_{\alpha_{i-1}}$, use Lemma $A_{\alpha_{1}}$ to define a homeomorphism $h_{i}$ of $E^{n}$ onto itself such that (a) $h_{i}(x)=x$ if $x \in B_{\alpha_{1}} \cup\left[E^{n}-S\left(B_{\alpha_{i-1}}, \varepsilon / 2^{i}\right)\right]$, (b) $h_{i}$ is locally semilinear on $E^{n}-B_{\alpha_{i-1}}$, (c) $h_{i} h_{i-1} \cdots h_{1}(C)$ is locally polyhedral except at the points of the $\alpha_{i}$ th derived set of $B_{\alpha_{i-1}}$, and (d) $\rho\left(x, h_{i}(x)\right) \leqq \min \left\{\varepsilon / 2^{i}, \varepsilon \cdot \rho(x, F) / 3^{i}, \rho\left(x, B_{\alpha_{0}}\right) / 4^{i}\right\}$. Notice that $\left(B_{\alpha_{i-1}}\right)_{\alpha_{i}}=B_{\alpha_{i-1}+\alpha_{i}} \subset B_{\alpha_{i}}$ since $\alpha_{i-1}+\alpha_{i} \geqq \alpha_{i}$, so that $h_{i} \cdots h_{1}(C)$ is locally polyhedral except possibly at the points of $B_{\alpha_{i}}$. Finally define $h(x)=\lim _{i \rightarrow \infty} h_{i} \cdots h_{1}(x)$ for each $x \in E^{n}$. The map $h$ is well-defined because $h_{i}$ moves no point more than $\varepsilon / 2^{i}$ and $\sum_{i=1}^{\infty} \varepsilon / 2^{i}=\varepsilon<\infty$. Routine series calculations show that $\rho(x, h(x))$ $\leqq \min \left\{\varepsilon, \varepsilon \cdot \rho(x, F), \frac{1}{2} \rho\left(x, B_{\alpha_{0}}\right)\right\}$ so that condition (d) of Lemma $\mathrm{A}_{\alpha_{0}}$ is satisfied. It is obvious from the construction that $h$ satisfies condition (a) of Lemma $A_{\alpha_{0}}$.

To show that $h$ is continuous at each point of $E^{n}-B_{\alpha_{0}}$, consider an arbitrary point $x \in E^{n}-B_{\alpha_{0}}$. Since $\rho(x, h(x)) \leqq \frac{1}{2} \rho\left(x, B_{\alpha_{0}}\right)$, and since $\left\{\mathrm{Cl} S\left(B_{\alpha_{i-1}}, \varepsilon / 2^{i}\right\}_{1}^{\infty}\right.$ is a decreasing sequence of compact sets intersecting in $B_{\alpha_{0}}$, there exists an integer $j(x)$ such that $h(x) \in E^{n}-\mathrm{Cl} S\left(B_{\alpha_{j(x)-1}}, 1 / 2^{j(x)}\right)$. Therefore there is a neighborhood $U$ of $x$ such that $h=h_{j(x)} \cdots h_{2} h_{1}$ on $U$, so that $h$ is continuous at $x$, and is also locally semilinear at $x$ if $x \notin B_{0}$. It follows also, if $x \in C$, that $h(C)$ is locally polyhedral at $h(x)$, since $x \notin B_{j(x)-1}$, so that conditions (b) and (c) of Lemma $A_{\alpha_{0}}$ are satisfied. 
To see that $h$ is $1-1$ on $E^{n}$, consider any two distinct points $x$ and $y$ of $E^{n}$. If $x, y \in B_{\alpha_{0}}$, then $h(x)=x \neq y=h(y)$ since $h$ is the identity on $B_{\alpha_{0}}$. If $x \in B_{\alpha_{0}}$ and $y \in E^{n}-B_{\alpha_{0}}$, then $h(x)=x$ while $h(y) \in E^{n}-B_{\alpha_{0}}$ as above, so that $h(x) \neq h(y)$. Finally suppose that $x, y \in E^{n}-B_{\alpha_{0}}$. Then denote by $k$ the maximum of $j(x)$ and $j(y)$. It follows that $h(x)=h_{k} \cdots h_{1}(x)$ and $h(y)=h_{k} \cdots h_{1}(y)$, so that $h(x) \neq h(y)$ because the $h_{i}$ are homeomorphisms.

Now suppose that $z \in E^{n}-B_{\alpha_{0}}$ and choose an integer $k$ such that $z \in E^{n}-S\left(B_{\alpha_{k}}, \varepsilon / 2^{k+1}\right)$, so that $h_{i}(z)=z$ if $i>k$, and let $y=h_{1}^{-1} \cdots h_{k}^{-1}(z)$. Then $h(y)=z$ so that $h$ maps $E^{n}-B_{\alpha_{0}}$ onto itself. Since $h$ is the identity on $B_{\alpha_{0}}$, it follows that $h$ maps $E^{n}$ onto $E^{n}$.

In order to prove that $h$ is a homeomorphism of $E^{n}$ onto itself, it remains to prove that $h$ is continuous at each point of $B_{\alpha_{0}}$ ( $h$ being the identity except on a compact set). Since $h$ is the identity on $B_{\alpha_{0}}$, it suffices to show that, given a point $x \in B_{\alpha_{0}}$ and an arbitrary neighborhood $U$ of $x$, there is a neighborhood $V$ of $x$ such that $h(V) \subset U$. First choose $\gamma>0$ such that $S(x, \gamma) \subset U$, and let $V=S(x, 2 \gamma / 3)$. Then, given $y \in V, \rho\left(y, B_{\alpha_{0}}\right)<2 \gamma / 3$ and $\rho(y, h(y)) \leqq \frac{1}{2} \rho\left(y, B_{\alpha_{0}}\right)<\gamma / 3$ so that $\rho(x, h(y)) \leqq \rho(x, y)+\rho(y, h(y))<2 \gamma / 3+\gamma / 3=\gamma$, and hence $h(y) \in S(x, \gamma) \subset U$. Thus $h(V) \subset U$. Therefore $h$ is continuous at $x$.

Consequently $h$ is a homeomorphism of $E^{n}$ onto itself satisfying the conditions of Lemma $A_{\alpha_{0}}$. It now follows by transfinite induction that Lemma $A_{\alpha}$ holds for each countable ordinal number $\alpha$. This completes the proof of the Theorem.

COROLlaRY. If the simple closed curve $C$ in $E^{n}, n>3$, is wild, then $C$ fails to be locally polyhedral at each point of some Cantor set.

Proof. If $B$ denotes the set of points at which $C$ is not locally polyhedral then, by the Theorem, $B$ is uncountable. As in the proof of the Theorem, choose a countable ordinal number $\alpha$ such that $B_{\beta}=B_{\alpha}$ if $\beta>\alpha$. Then $B_{\alpha}$ is an uncountable compact perfect set, at each point of which $C$ fails to be locally polyhedral. If $B_{\alpha}$ is totally disconnected, then it is a Cantor set. Otherwise $B_{x}$ contains an arc, which in turn contains a Cantor set.

\section{REFERENCES}

1. W. A. Blankinship, Generalization of a construction of Antoine, Ann. of Math. (2) 53 (1951), 276-297.

2. R. Fox and E. Artin, Some wild cells and spheres in 3-dimensional space, Ann. of Math. (2) 49 (1948), 979-990.

3. V. K. A. M. Gugenheim, Piecewise linear isotopy and embedding of elements and spheres (I), Proc. London Math. Soc. 3 (1953), 29-53.

4. W. Sierpinski, General topology, Univ. of Toronto Press, Toronto, 1952.

5. E. C. Zeeman, Unknotting spheres, Ann. of Math. (2) 72 (1960), 350-361.

UNIVERSITY OF TENNESSEE,

KNoXville, TenNessee

UNIVERSTTY OF WISCONSIN,

MAdison, Wisconsin 